\title{
PLEIADES-HR INNOVATIVE TECHNIQUES FOR RADIOMETRIC IMAGE QUALITY COMMISSIONING
}

\author{
Gwendoline BLANCHET, Laurent LEBEGUE, Sébastien FOUREST, Christophe LATRY, \\ Florence POREZ-NADAL, Sophie LACHERADE, Carole THIEBAUT \\ CNES 18, avenue Edouard Belin, 31401 TOULOUSE CEDEX 4 France \\ Phone: 33.(0)5.61.27.48.59 Fax: 33.(0)5.61.27.31.67 E-mail: gwendoline.blanchet@cnes.fr
}

ISPRS and IAA : Pléiades Inflight Calibration and Performance Assessment

KEY WORDS: Image Quality, Radiometry, Calibration

\begin{abstract}
:
The first Pleiades-HR satellite, part of a constellation of two, has been launched on December 17, 2011. This satellite produces high resolution optical images. In order to achieve good image quality, Pleiades-HR should first undergo an important 6 month commissioning phase period. This phase consists in calibrating and assessing the radiometric and geometric image quality to offer the best images to end users. This new satellite has benefited from technology improvements in various fields which make it stand out from other Earth observation satellites. In particular, its best-in-class agility performance enables new calibration and assessment techniques. This paper is dedicated to presenting these innovative techniques that have been tested for the first time for the PleiadesHR radiometric commissioning.

Radiometric activities concern compression, absolute calibration, detector normalization, and refocusing operations, MTF (Modulation Transfer Function) assessment, signal-to-noise ratio (SNR) estimation, and tuning of the ground processing parameters. The radiometric performances of each activity are summarized in this paper.
\end{abstract}

\section{INTRODUCTION TO PLEIADES-HR SATELLITE}

The PLEIADES program is a space Earth Observation system led by France, under the leadership of the French Space Agency (CNES). It will operate in 2012 two agile satellites designed to provide optical images to civilian and defence users.

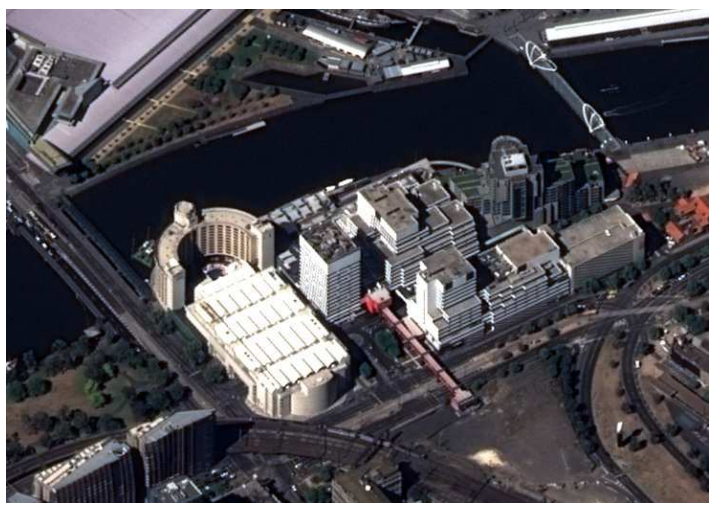

Figure 1. Pleiades-HR image of Melbourne downtown, (c) CNES Copyright 2012

Since it was successfully launched on December 17, 2011, Pleiades 1A high resolution optical satellite has been thoroughly tested and validated during the commissioning phase led by CNES [1].

One of the major improvements of this satellite is its platform agility. It allows the satellite to move quickly from one attitude to another, enabling rapid collection of separated point targets and an increase of imaging capacity. Thanks to this agility, several guidance strategies have been defined in order to facilitate both the radiometric and geometric satellite commissioning. They offer new possibilities of measuring the performance and lower operational costs by avoiding some specific image campaigns. This paper is dedicated to the radiometric part. For more information about the geometric part, please refer to [2].

Images are simultaneously acquired in Panchromatic (PA) and multi-spectral (XS) mode, which allows, in nadir acquisition condition, to deliver $20 \mathrm{~km}$ wide, false or natural coloured scenes with a $70 \mathrm{~cm}$ ground sampling distance after PA+XS fusion [3]. The scan-line is comprised of 5 detector ChargeCoupled Device (CCD) arrays for PA and XS bands with overlapping Inter-Array-Zone (IAZ) to ensure line continuity. These arrays are located in the focal plane of the telescope and acquire a scan-line over an integration time (push-broom principle) [4]. Because of distortion and PA TDI (Time Delay Integration) tilts, the on-ground projections of PA and XS lines of sight are not straight lines. Consequently, some guidances are adapted to the considered band (PA or XS). For the PA band, the TDI enables to achieve five different exposure levels.

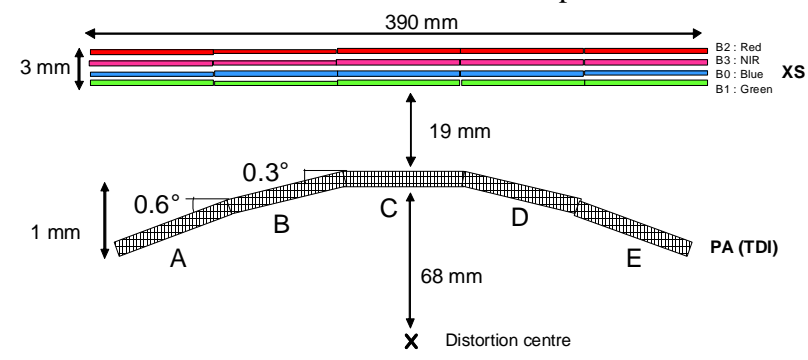

Figure 2. Pleiades-HR focal plane 


\section{RADIOMETRIC ACTIVITIES}

\subsection{Inter-detector normalization and compression}

Because of the non uniform detector sensitivity along the array, the raw image of a uniform landscape is striped vertically. Interdetector normalization consists in applying a pixel-to-pixel gain and offset so that the output image is uniform.

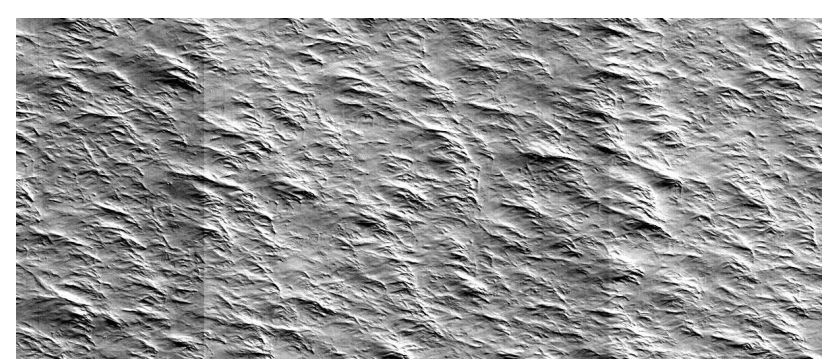

Figure 3. Raw PA Pleiades-HR image of Antarctic, vertical streaks are visible, (C) CNES Copyright 2012

A pre-launch full calibration has showed that the detector response has a bilinear response as a function of the input radiance $\mathrm{L}$, unlike most satellites which have a linear response (Quickbird, WorldView-1 [5], Spot5, ...).

The bilinear model is:

$$
\begin{array}{cc}
X(n, b)=A \gamma_{1}(b) \cdot g_{1}(n, b) L+C(n, b) & \text { if } L \leq L_{s}(n, b) \\
X(n, b)=A\left\{\gamma_{2}(b) \cdot g_{2}(n, b) \cdot\left[L-L_{s}(n, b)\right]+\gamma_{1}(b) \cdot g_{1}(n, b) L_{s}(n, b)\right\}+C(n, b) & \text { otherwise }
\end{array}
$$

for each detector $\mathrm{n}$ of register $\mathrm{b}$, where

$\mathrm{A}$ is the absolute calibration factor linking radiance measurement to the pixel bit-count;

- $\quad \mathrm{C}$ are the dark currents classically estimated on ocean images acquired on night orbits (ascending). Thanks to Pleiades agility, these dedicated images can be replaced by star images (intrinsically mainly dark) already available with the MTF theme;

$\gamma$ and $\mathrm{g}$ are the relative offsets estimated either with uniform landscape image acquisition campaign (classical method, images of well-known uniform landscapes such as Antarctic and Greenland are acquired routinely during the first six months in orbit) or with dedicated AMETHIST images (new method).

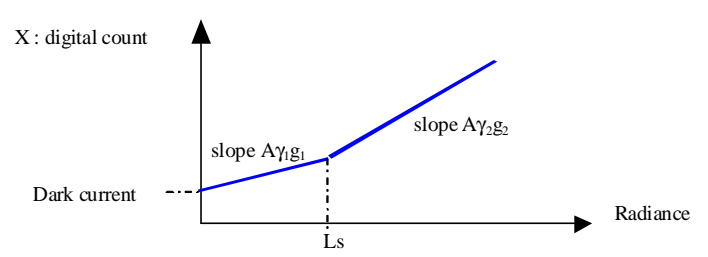

Figure 4. Bilinear radiometric model

The values of inter-detector normalization coefficients depend on the number of integration lines used in the TDI. This number has been set to 13 during the first commissioning months. The possible values are $[7,10,13,16,20]$. Some tests will be run until the end of the commissioning to select the best number of
TDI lines for operational needs. A high number ensures a good signal to noise ratio if the smearing effect is small enough, whereas a small number ensures that no saturation will occur. Since images are collected at a dynamic range of 12 bits per pixel, the saturation value is 4095 .

Inter-detector normalization can be done either on-ground as a post-processing or on-board as normally expected. The nonlinear normalization coefficients, called On-Board Calibration Data (OBCD) are transmitted from the ground to the satellite and are updated as often as necessary. Most images (except the ones used for the estimation of these coefficients) are radiometrically corrected on-board with the calibration factors. Images are then compressed and transmitted to the ground. The compression algorithm [6] is based on a 3 decomposition level wavelet transform similar to JPEG2000 standard and adapted to satellite imagery: it performs a so-called "strip-based" compression suited for push-broom high data rate imagers. In this mode, the same bit rate is allocated to each 16 lines image block. The bit rate can be chosen among the following values: $[2,2.22,2.5,2.86,3.33,4] \mathrm{bits} /$ pixel. Since the bit stream is embedded, images with low bit rates can be simulated from images with the highest bit rate (4 bits/pixel). During commissioning, the nominal on-board compression bit rate has been optimized to minimize both the compression artefact ("butterfly" pattern or excessive blur) and the bit rate.

In the end, the initial recommended bit rates have been set to 2.86 for PA band and 3.33 for XS bands.

The two main reasons why it is preferable to do the normalization before the on-board compression are the following:

- compression disturbs the radiometric model of each detector since it mixes information from different image columns;

- streaks coding is unnecessary and wastes the bit budget when the output image should be a constant.

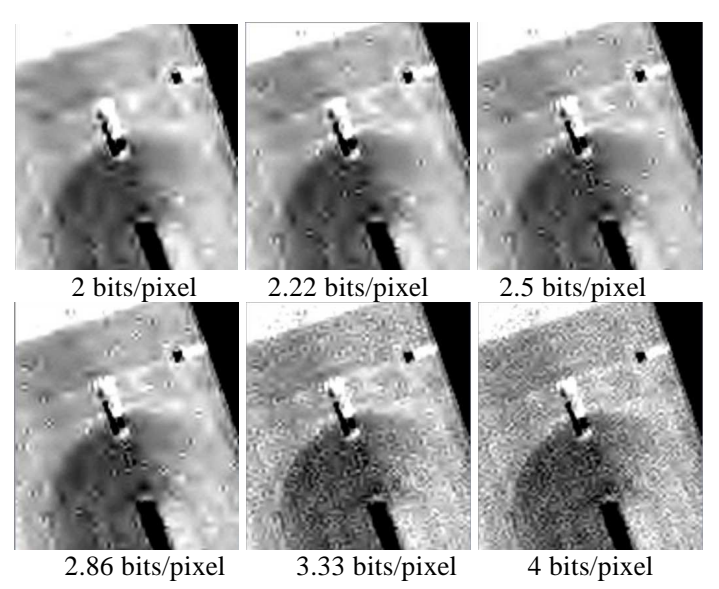
Figure 5. Restored PA Pleiades-HR image of Melbourne acquired at 4 bits/pixels and simulated at lower bit rates, (C) CNES Copyright 2012 


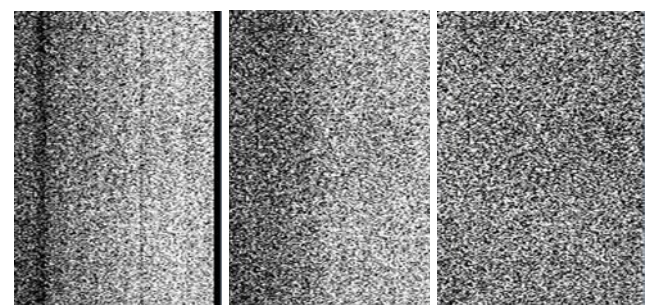

Figure 6. (a) Raw Antarctic image collected on orbit, high (HF) and low (LF) frequency vertical streaks are visible (b) Raw image radiometrically corrected with the pre-launch calibration factors: some streaks are still visible, especially LF ones (c) Raw image radiometrically corrected with on-orbit updated calibration factors. Here the streaks are no longer visible.

(c) CNES Copyright 2012

To estimate the inter-detector gains, each detector must receive the same radiance. The classical way uses uniform images acquired at the reference radiances (L1, L2, L3) [7].

\begin{tabular}{|c|c|c|c|c|c|}
\hline & PA & B0 & B1 & B2 & B3 \\
\hline L2 & 100 & 130 & 115 & 100 & 90 \\
\hline \multicolumn{6}{|c|}{ Table 1. Reference L2 radiance $\left(\mathrm{W} / \mathrm{m}^{2} / \mu \mathrm{m} / \mathrm{sr}\right)$}
\end{tabular}

But this approach is limited since pure uniform images do not exist on Earth (landscape residue may remain and clouds may degrade the image). The AMETHIST method [7] has been tested for the first time on Pleiades. This efficient method avoids the uniformity constraint. A special guidance has been designed to ensure that each detector sees the same target landscape line: the ground projection of the scan-line is aligned to the ground velocity. The push-broom principle has been diverted from its original goal to produce $20 \mathrm{~km}$ swath images by creating very narrow (few meters) swath striped images. The AMETHIST images are made of diagonal stripes, each stripe is associated with the same ground point and its length indicates the number of detectors that have seen the point.

The geometric constraint (the detector must see the same landscape area) cannot be achieved simultaneously for both the PA and XS bands due to the focal plane configuration. Hence, those bands have different curved attitude guidance.

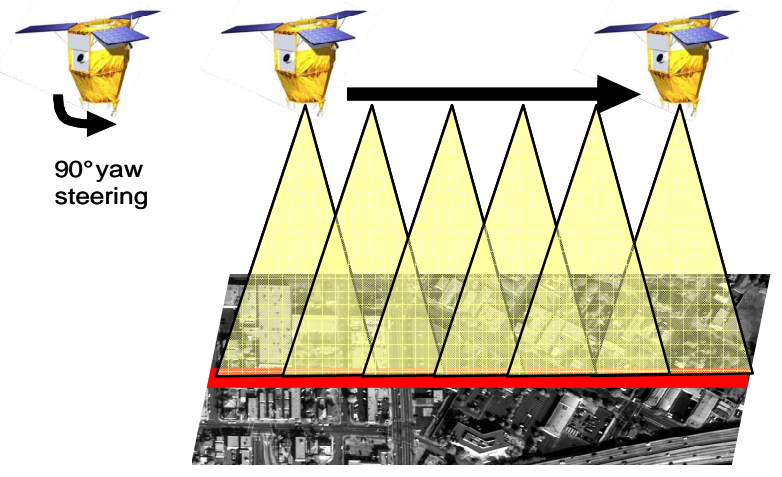

Figure 7. AMETHIST acquisition principle (in red: target landscape line)

After a pre-processing that globally shifts each column of the raw image, we get an image that contains all needed information. This means that every row contains the set of detector responses to the same landscape. Thus, non-linear normalization coefficients can be computed by a histogram matching method.

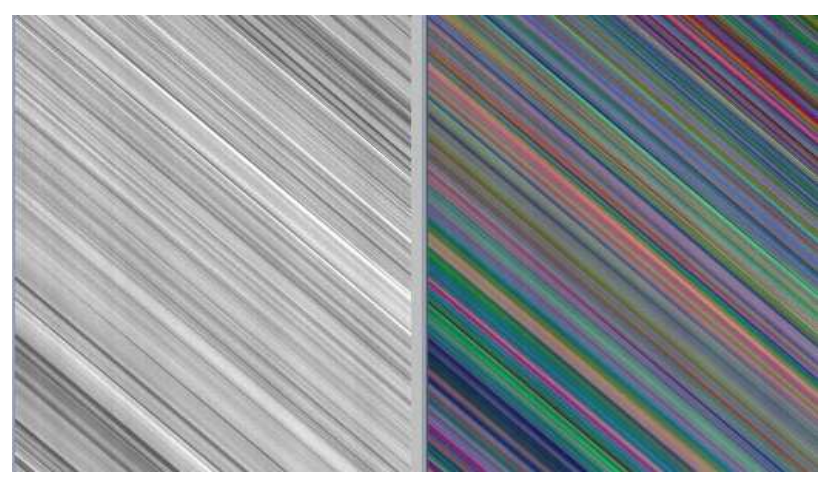

Figure 8. Example of AMETHIST PA and XS images (C) CNES Copyright 2012

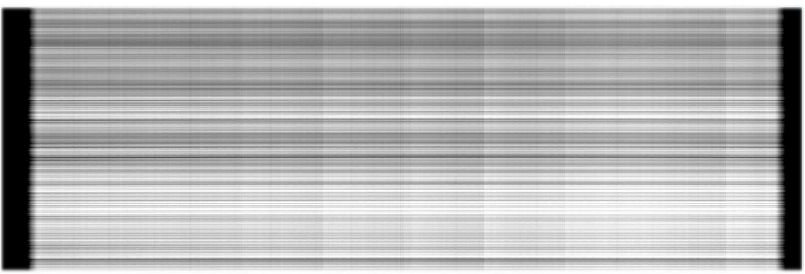

Figure 9. Example of rectified AMETHIST PA image (central array, IAZ are visible on both sides)

This method requires few acquisitions (typically 30 images) to fulfil the normalization coefficient estimation without weather and site constraints. It is however recommended to perform several acquisition on various sites to cover a large grey level dynamics with flat relief. The coefficients update frequency is expected to be 6 months.

The temporal evolution of the detector response has been analysed on-ground since 2009. It showed noticeable changes that should be monitored after launch. The NeDLI measures the residual non-uniformity after radiometric correction (a high NeDLI value indicates that vertical streaks are visible), so this is how the detector evolution is detected. On-ground measurements allowed to recommend a coefficient update every 6 months. The first four months showed that the NeDLI evolved slowly enough to maintain the 6 months prediction.

\begin{tabular}{|c|c|c|c|c|}
\hline PA & B0 & B1 & B2 & B3 \\
\hline+0.08 & +0.15 & +0.2 & +0.3 & +0.3 \\
\hline
\end{tabular}

Table 2. NeDLI evolution during the first 3 months (estimation is done outside IAZ) (assessed April 5, 2012).

\subsection{Absolute calibration}

The estimation of the absolute calibration coefficients for every spectral band is done on various types of landscapes to achieve cross method comparison and temporal monitoring [8]. Input images are radiometrically corrected with inter-detector normalization. Those landscapes have been defined over the years thanks to previous satellite commissioning. They are now fully characterized and are still used by other CNES in-orbit satellites which allows inter-calibration as well. 
These landscapes include:

uniform landscapes: sand and snow desert;

- La Crau test site equipped with the CIMEL radiometer providing automatic measurement of multi-angular viewing of atmosphere and close neighbouring ground reflectance;

- the Moon.

This large range of methods allows to guarantee a 5\% accuracy of the absolute calibration coefficient estimation. First results showed that the measurements are very close to the pre-launch values.

Thanks to Pleiades agility, some innovative studies are undertaken to study the sensitivity of the absolute calibration coefficient measurement with respect to the satellite viewing angle. To do so, the video sequences of some sites have been acquired.

\subsection{MTF assessment and refocusing operations}

The refocusing system on Pleiades consists of a thermal control of the telescope's secondary mirror position. Some new methods have been developed and tested that use the defocusing ability of the instrument on a large range of values (typically, a 15 minute pause is carried out after each thermal control change for thermal stabilization).

Thanks to Pleiades agility, a new method based on stars acquisition has been developed and tested [9]. Those point-like objects are very interesting since they allow a direct computation of the MTF (by considering a large number of stars and by interlacing each elementary response to produce a well sampled one). This method offers tremendous advantages compared to former methods:

- $\quad$ No dependency on weather condition (astral objects);

- No conflict with other Earth observation acquisitions (low operational impact).

Thanks to these very efficient methods, the instrument was refocused 5 days after launch in order to increase the PA MTF value at Nyquist frequency. These MTF values (of both XS and PA bands) have been taken into account in the Image Quality performance assessment methods, such as the restoration algorithm [10] or the noise measurement.

\subsection{Signal-to-noise ratio assessment}

Pleiades instrument noise model $\sigma_{\mathrm{c}}$ (or column) corresponds to a quadratic sum of the various contributors: photon noise $\sigma_{\mathrm{s}}$, quantization noise $\sigma_{\mathrm{q}}$, dark noise and electronic noise $\sigma_{\mathrm{obs}}$ : $\sigma_{c}=\sqrt{\sigma_{o b s}^{2}+\sigma_{q}^{2}+\sigma_{s}^{2}}$

The model is expressed either at the end of the imaging chain as a digital count $\mathrm{q}$ or at the instrument input in units of radiance $\left(\mathrm{W} / \mathrm{m}^{2} / \mathrm{sr} / \mu \mathrm{m}\right)$. Conversion from one to the other is ensured by means of the absolute calibration coefficient A.

The overall column noise model is thus written as $\sigma_{c}(L)=\sqrt{a_{L}+b_{L} L}$, or equivalently $\mathrm{V}(\mathrm{L})=\mathrm{a}_{\mathrm{L}}+\mathrm{b}_{\mathrm{L}} \cdot \mathrm{L}$.

In digital count unit, the model is $\mathrm{V}(\mathrm{q})=\mathrm{a}+\mathrm{b}$.q, where $\mathrm{a}$ and $\mathrm{b}$ are the two parameters to estimate. Parameter a does not depend on the input radiance, it can be estimated with the dark current images.

\begin{tabular}{|c|c|c|c|c|c|}
\hline & PA & B0 & B1 & B2 & B3 \\
\hline $\mathrm{a}$ & 5.14 & 3.24 & 2.61 & 2.54 & 2.28 \\
\hline
\end{tabular}

Table 3. Estimation of a (assessed April 5, 2012).

Several methods have been used for the estimation of the instrument noise, two of them are brand new. Both methods were successfully tested during commissioning to retrieve ground measurements.

\subsubsection{Steady-mode acquisition}

The first method takes benefit of the satellite agility thanks to the "slow-motion" guidance. A steady-mode acquisition has been defined to acquire the target ground points. The principle is to steer the satellite so that the projection of the scan-line on the ground remains constant along the image.

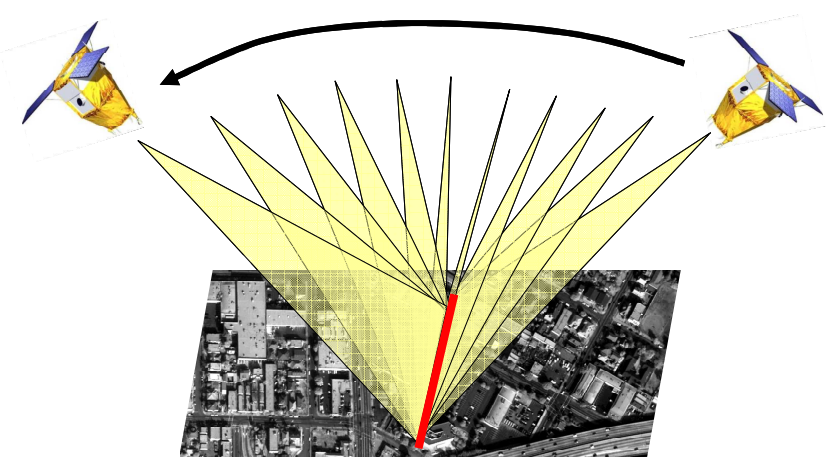

Figure 10. Steady-mode acquisition principle (in red: target landscape line)

Therefore, each elementary detector acquires the same point on the ground along the column-wise direction with a slight change in the viewing direction. An example of the obtained image is shown in Fig.11, where it becomes easy to compute the temporal evolution of each elementary detector response for a large set of input radiance. Indeed each column corresponds to the temporal response of one detector for a given input radiance.

Because of possible geometric distortion evolution between the beginning and the end of the image due to projection effect of the line of sight, the temporal response of each detector is analysed on non-overlapping 50 pixels length column blocks.

In the end, the global noise model is obtained by combining the response of all the detectors, hence covering a large input radiance. In order to increase the input radiance range, several "slow-motion" images are combined. Fig.12 shows an example with 32 input images. In the end, we can assess the instrumental noise model and its contribution to the radiometric signal-tonoise ratio budget. 


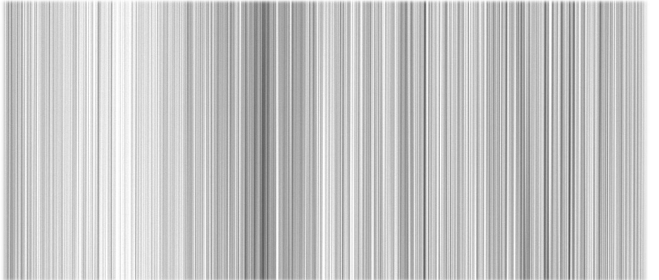

Figure 11. Example of slow-motion image (c) CNES Copyright 2012

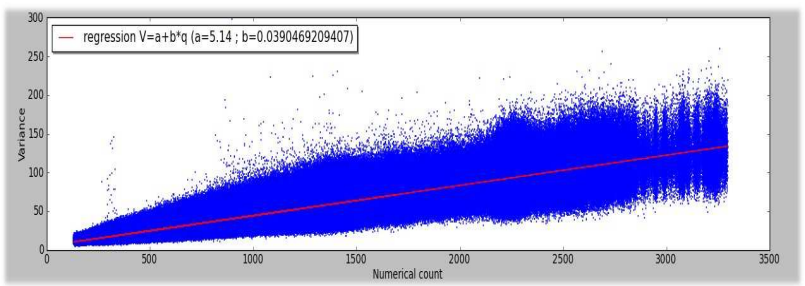

Figure 12. Estimation of parameter $\mathrm{b}$ for the PA band

\begin{tabular}{|c|c|c|c|c|c|}
\hline & PA & B0 & B1 & B2 & B3 \\
\hline b & 0.039 & 0.037 & 0.041 & 0.043 & 0.047 \\
\hline
\end{tabular}

Table 4. Estimation of b (assessed April 5, 2012).

\subsubsection{High-frequency analysis}

Another new technique called BETSI [11] consists in measuring high frequency residues after high-frequency filtering with the hypothesis that high spatial frequencies are sufficiently weakened by the MTF so that only noise remains near Nyquist frequency.

There are three possible ways to satisfy this hypothesis:

a low MTF value at Nyquist frequency;

- acquisition of a landscape with low complexity (for example, urban areas should be avoided since they contain HF);

acquisition of a defocused image (by using the thermal control of the telescope's secondary mirror position).

Since Pleiades has high MTF value at Nyquist frequency and since the BETSI technique should normally have a low operational impact and thus should not require specific landscape acquisitions, the method has been applied on defocused PA images.
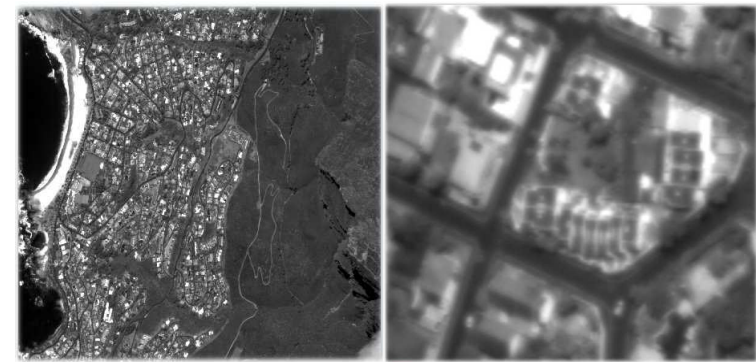

Figure 13. Example of Cape Town defocused image. Thermal control $=32^{\circ} \mathrm{C}$. Acquired on Dec 23, 2011
The mathematical tool used in BETSI may be Fourier transform (FT) or wavelet packet decomposition. The idea is to select a high frequency subset where landscape components are sufficiently weakened to be insignificant when compared to noise. The method based on the FT consists in taking the FT of the image, applying a frequency binary mask in order to select the chosen HF domain and then coming back to the spatial domain thanks to the inverse FT, which gives a HF noise image. Both original and filtered images are tiled in non overlapping patches of size $(\mathrm{N}, \mathrm{N})$. For each tile, the mean signal value from the original image and the variance from the HF noise image. Since noise variance is computed from a subset of the Fourier domain, it must be amplified by a normalization factor, which is merely the ratio of the surface of the subset to the surface of the whole frequency domain. One image produces thus a large collection of (local signal q, noise variance V) couples, allowing a statistically correct estimation of the linear function $\mathrm{V}(\mathrm{q})=\mathrm{a}+\mathrm{b} \cdot \mathrm{q}$.

The frequency domain can be chosen manually (by fixing for example the radius of a binary circle mask) or automatically (by letting the algorithm find the best zone). The method produces all the more robust noise estimation that the subset of the Fourier domain is big.

When the MTF is known (this is the case for the Pleiades commissioning see Fig.14), the frequency domain can be easily chosen manually in the reciprocal cell normalized (the reciprocal cell is the frequency zone associated with the spatial sampling grid).

For example, in the Pleiades defocused PA case of Cape Town (Fig.13), a radius of 0.65 is satisfying since the MTF is close to 0 from the Nyquist frequency / 2 to the Nyquist frequency. So, noise can be estimated from a large subset of Fourier domain, which ensures a good estimation.

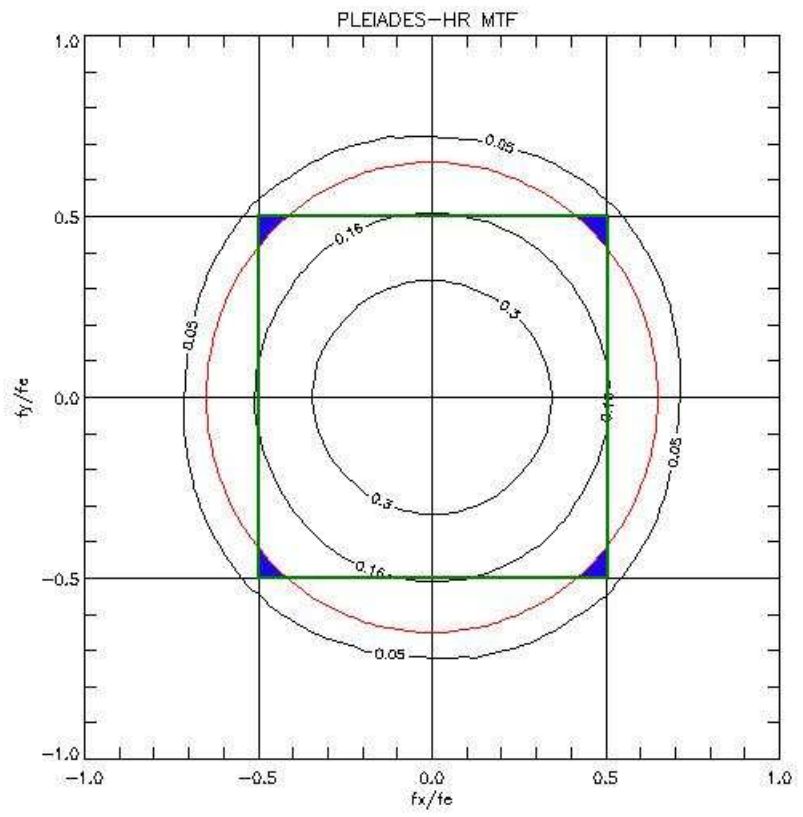

Figure 14. Level lines of the PA Pleiades MTF (in black). Axis fx (resp. fy) represents the frequency along $\mathrm{X}$ and $\mathrm{Y}$ directions.

Reciprocal cell (in green). At Nyquist frequency $(\operatorname{abs}(\mathrm{fx} / \mathrm{fe})=\operatorname{abs}(\mathrm{fy} / \mathrm{fe})=0.5)$, the MTF is equal to 0.16 . 
The red line represents the radius 0.65 of the mask, and the blue zone indicates the associated frequency subset. In this zone, $\max (\mathrm{MTF})=0.0987, \min (\mathrm{MTF})=0.0554, \operatorname{mean}(\mathrm{MTF})=0.079$.

For defocused images, the MTF is equal to zero in the frequency domain $[-0.5:-0.25,0.25: 0.5]$, which ensures that the blue zone contains only noise.

\section{MAIN RADIOMETRIC IMAGE QUALITY PERFORMANCE}

The table below gives the main radiometric image quality performance of Pleiades 1A assessed between the launch and April 5, 2012. Some of them will be updated by the end of the commissioning phase scheduled for June 30, 2012.

\begin{tabular}{|c|l|l|}
\hline \multicolumn{2}{|c|}{ Image Quality criteria } & $\begin{array}{c}\text { Perfor } \\
\text { mance }\end{array}$ \\
\hline \multirow{2}{*}{$\begin{array}{c}\text { NeDLI } \\
\text { evolution } \\
\text { (first 3 } \\
\text { months })\end{array}$} & PA & +0.08 \\
\cline { 2 - 3 } & B0 & +0.15 \\
\cline { 2 - 3 } & B1 & +0.2 \\
\cline { 2 - 3 } & B2 & +0.3 \\
\hline \multirow{2}{*}{ Abs. } & Bbsolute calibration accuracy & +0.3 \\
\hline \multirow{3}{*}{ FTM } & PA MTF @ Nyquist frequency & $5 \%$ \\
\cline { 2 - 3 } & XS MTF @ Nyquist frequency (X axis) & 0.16 \\
\cline { 2 - 3 } & XS MTF @ Nyquist frequency (Y axis) & 0.33 \\
\hline \multirow{3}{*}{ SNR } & PA SNR @ L2 & 150 \\
\cline { 2 - 3 } & B0 SNR @ L2 & 174 \\
\cline { 2 - 3 } & B1 SNR @ L2 & 151 \\
\cline { 2 - 3 } & B2 SNR @ L2 & 136 \\
\cline { 2 - 3 } & B3 SNR @ L2 & 142 \\
\hline
\end{tabular}

Table 5. Main Image Quality performance. Preliminary results (assessed April 5, 2012).

\section{ACKNOWLEDGEMENTS}

The author would like to thank the whole team of image experts who have performed a great job, spending time, energy and enthusiasm during the 6 months of the image quality commissioning. He would also like to thank the Pleiades image ground processing team for its support and especially the ICC (Image Calibration Centre) exploitation and Pleiades Mission teams for their constant good mood, providing the experts with acquisitions and image products.

\section{REFERENCES}

[1] L. Lebègue \& al, "Pleiades-HR Image Quality Commissioning”, ISPRS Melbourne 2012.

[2] D. Greslou \& al, "Pleiades-HR innovative techniques for Geometric Image Quality Commissioning", ISPRS Melbourne 2012.

[3] A. Gleyzes \& al, "Pleiades system architecture and main performances", ISPRS Melbourne 2012.
[4] F. deLussy \& al, "Pleiades HR in flight geometrical calibration: Localisation and mapping of the focal plane", ISPRS Melbourne 2012.

[5] K. Krause, "WorldView-1 pre and post-launch radiometric calibration and early on-orbit characterization", SPIE 2008.

[6] C. Thiebaut \& al, "Improving a DWT-based compression algorithm for high image-quality requirement of satellite", SPIE 2011.

[7] Ph. Kubik \& al, "AMETHIST: A MEthod for Equalization Thanks to HISTograms", SPIE Maspalomas 2004.

[8] S. Lachérade \& al, "Pleiades absolute calibration: first results", ISPRS Melbourne 2012.

[9] S. Fourest \& al, "Star-based methods for Pleiades HR commissioning”, ISPRS Melbourne 2012.

[10] C. Latry \& al, "Restoration technique for Pleiades-HR panchromatic images”, ISPRS Melbourne 2012.

[11] C. Latry \& al, "Geometric/radiometric calibration from ordinary images for high resolution satellite", SPIE 2011. 